\title{
INMUNOTERAPIA EN CÁNCER: DE LOS INICIOS AL PREMIO NOBEL
}

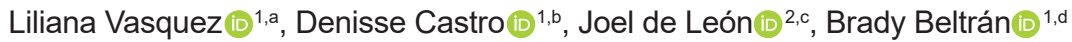 \\ ${ }^{1}$ Centro de Investigación de Medicina de Precisión, Instituto de Investigación, Facultad de Medicina Humana, \\ Universidad de San Martín de Porres, Lima, Perú. \\ 2 Centro de Investigación en Infectología e Inmunología, Facultad de Medicina Humana, Universidad de San Martín \\ de Porres, Lima, Perú. \\ ${ }^{a}$ Médico oncólogo pediatra; ${ }^{\mathrm{b}}$ médico oncóloga; ${ }^{\mathrm{c}}$ Licenciado en Bioquímica, Doctor en Ciencias Biológicas; ${ }^{\mathrm{d}}$ médico \\ oncólogo, magister en Biología molecular.
}

\section{RESUMEN \\ La inmunoterapia ha significado un gran avance científico en el tratamiento del cáncer en los últimos años, siendo considerada como la piedra angular terapéutica de algunas neoplasias. Sin embargo, en algunos tipos de cáncer sólo una fracción de pacientes alcanza beneficio, planteando los desafíos y limi- tantes que nos llevan a la necesidad de entender la complejidad de la biología tumoral, el microambiente tumoral y la capacidad de respuesta de cada agente. Los resultados superan actualmente lo alcanzado por la quimioterapia convencional, aunque aún no se puede precisar si estas respuestas son duraderas o representan curación. En la presente revisión, se propone un enfoque amplio sobre la inmunoterapia en cáncer, desde los conceptos básicos hasta su aplicación clínica, descubrimiento que fue galardonado con el Premio Nobel de Medicina en el 2018. \\ Palabras clave: Inmunoterapia; Neoplasias; Oncología Médica (fuente: DeCS BIREME). \\ CANCER IMMUNOTHERAPY: FROM INCEPTION TO NOBEL PRIZE}

\begin{abstract}
Immunotherapy has meant a great scientific advance in the treatment of cancer in recent years, being considered as the therapeutic cornerstone of some neoplasms. However, in some types of cancer only a fraction of patients achieve benefit, posing the challenges and limitations that lead us to the need to understand the complexity of tumor biology, the microenvironment and the responsiveness of each agent. The results currently exceed what was achieved by conventional chemotherapy, although it is not yet possible to determine whether these responses are lasting or represent cure. In this review, a broad approach to immunotherapy in cancer is proposed, from the basics to its clinical application, a discovery that was awarded the Nobel Prize in Medicine in 2018.
\end{abstract}

Keywords: Immunotherapy; Neoplasms; Medical Oncology (source: MeSH NLM).

\section{INTRODUCCIÓN}

La inmunoterapia se está convirtiendo en la piedra angular de los enfoques terapéuticos en pacientes con cáncer, cuyo éxito radica en manipular la actividad del sistema inmunológico, tanto de las células como de los mediadores moleculares con el fin de aniquilar las células malignas, mientras las células normales del organismo permanecen intactas. Estas terapias

Citar como: Vasquez L, Castro D, De León J, Beltrán B. Inmunoterapia en cáncer: de los inicios al premio Nobel. Rev Peru Med Exp Salud Publica. 2020;37(1):115-21. Doi: https://doi. org/10.17843/rpmesp.2020.371.4329

Correspondencia: Brady Beltran Garate; bgbrady@hotmail.com

Recibido: $07 / 03 / 2019$

Aprobado: 15/01/2020

En línea: 19/03/2020 han demostrado inducir la regresión tumoral completa y duradera en algunos tipos de cáncer, convirtiéndose un verdadero hito en las terapias dirigidas, incluso en el contexto de enfermedades avanzadas o metastásicas ${ }^{(1)}$.

La presente revisión propone un enfoque amplio sobre la inmunoterapia en cáncer, desde los conceptos básicos hasta su aplicación clínica. La misma se fundamenta principalmente en el descubrimiento por los científicos pioneros James P. Allison y Tasuku Honjo, ganadores del Premio Nobel en Medicina en el 2018, de los puntos de control de la respuesta inmune CTLA4 (del inglés, cytotoxic T-lymphocyte antigen 4) y el eje PD-1/PDL-1 (del inglés, programmed death-1 y programmed death-1 ligand), respectivamente. Estas moléculas son actualmente blancos clave de la inmunoterapia en cáncer. 
Para la selección de los trabajos incluidos en esta revisión se realizó una búsqueda, en Pubmed, de trabajos originales y revisiones temáticas, publicados después del 2010 sobre inmunooncología e inmunoterapia en cáncer. Se excluyeron de esta búsqueda aquellos trabajos de los que solo se pudo revisar el resumen. Considerando el gran volumen de información en este campo, se realizó una selección de aquellos estudios de mayor trascendencia por su relevancia teórico-práctica y que resultan referencia obligada en este campo.

\section{INMUNO-ONCOLOGÍA: DE INMUNOEDITARA INMUNOVIGILAR}

En el 2011, las evidencias experimentales y clínicas acumuladas acerca de la biología de las células neoplásicas le permitieron a los investigadores Douglas Hanahan y Robert Weinberg fundamentar ocho propiedades esenciales que aseguran la progresión del cáncer: señales proliferativas constitutivas, evasión de señales antiproliferativas, resistencia a la apoptosis, potencial replicativo sostenido, inducción de angiogénesis, reprogramación metabólica, capacidad metastásica y capacidad para evadir el sistema inmune ${ }^{(2,3)}$. Estas cualidades se sustentan en la inestabilidad genética de las células neoplásicas y en el contexto inflamatorio crónico ligado a la progresión tumoral.

Las propiedades inherentes a las células neoplásicas les permiten evadir los mecanismos extrínsecos e intrínsecos que controlan la carcinogénesis. Dentro de los mecanismos extrínsecos se incluye la demostrada capacidad del sistema inmune de activar sus mecanismos efectores para controlar la progresión tumoral ${ }^{(4)}$. Este evento conceptualizado como inmunovigilancia transcurre en cuatro etapas principales ${ }^{(5)}$ (Figura 1): 1) la detección por las células presentadoras de antígenos (APC, del inglés, Antigen Presenting Cells) de señales de peligro producidas por la pérdida de la homeostasis en los tejidos donde se produce la carcinogénesis, así como por la presencia de antígenos específicos o asociados al tumor, lo cual produce una serie de cambios morfofuncionales en las células dendríticas e implica la maduración de estas células y su migración a los ganglios linfáticos adyacentes; 2) activación, proliferación y diferenciación funcional de los linfocitos $\mathrm{T}$ colaboradores $\mathrm{CD}^{+}$y citotóxicos $\left.\mathrm{CD}^{+} ; 3\right) \mathrm{mi}-$ gración de los linfocitos activados al tejido alterado y su extravasación para interactuar con las células transformadas, y 4) ejecución de los mecanismos efectores de la inmunidad que deben conducir a la eliminación de las células transformadas. En esta cuarta etapa, la muerte de las células neoplásicas incrementa la liberación de antígenos inmunogénicos.

En el concepto descrito, se incluyen tanto neoantígenos como otras moléculas que actúan como señales de peligro para el sistema inmune, las que son liberadas como consecuencia de la muerte masiva por apoptosis. Lo anterior poten-

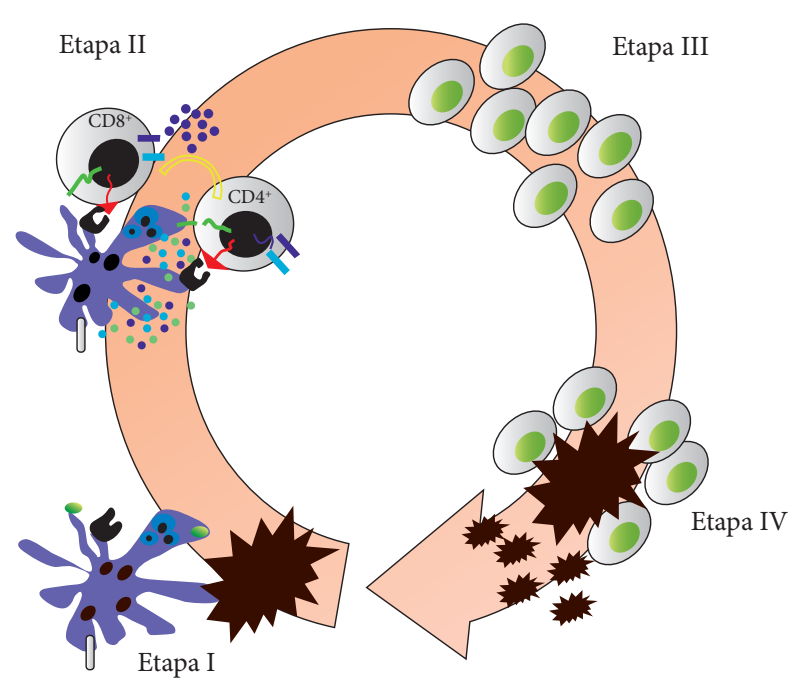

Figura 1. Etapas principales en que se establece y ejecuta la inmunovigilancia al cáncer. Etapa I: captura de antígenos tumorales y maduración de las células dendríticas; Etapa II: estimulación en los ganglios linfaticos adyacentes de la respuesta inmune mediada por linfocitos específicos; Etapa III: migración de los linfocitos activados al microambiente tumoral; Etapa IV: eliminación de las células tumorales por los mecanismos efectores de la inmunidad.

cia la estimulación de las APC, reiniciando un proceso cíclico virtuoso. La influencia de la inmunovigilancia en la progresión tumoral es tan relevante que se ha demostrado el valor pronóstico y predictivo de la composición y localización del infiltrado leucocitario en el microambiente tumoral ${ }^{(6-8)}$.

Como se mencionó anteriormente, la inestabilidad genética es una cualidad habilitante para las propiedades esenciales del cáncer ${ }^{(2,3)}$. Esta condición determina que en las neoplasias se produzca una evolución somática, de manera que los clones que guían la progresión tumoral son los de mayor capacidad de adaptación a las presiones selectivas que se producen en el microambiente tumoral ${ }^{(9)}$. Entre estas presiones selectivas se incluye la propia inmunovigilancia, de manera que la actividad antitumoral del sistema inmune edita la composición de los clones de células neoplásicas en el cáncer ${ }^{(10)}$. Lo anterior enriquece al tumor con células neoplásicas con capacidad de evadir la inmunovigilancia, lo que evidencia el papel dual del sistema inmune sobre la progresión tumoral. Este efecto de edición clonal se ha nombrado inmunoedición, concepto que refleja de manera más integral la compleja relación entre el sistema inmune y el cáncer ${ }^{(10)}$.

La inmunoedición incluye tres etapas $\left.{ }^{(10)}: 1\right)$ eliminación, en la que se produce la inmunovigilancia al cáncer con la consecuente muerte de las células neoplásicas por la actividad del sistema inmune innato y adaptativo. El incremento en la frecuencia de neoplasias en modelos de experimentación y en pacientes con inmunodeficiencias sustenta la ocurrencia de esta etapa; 2) equilibrio, en la que los mecanismos efectores del sistema inmune pueden controlar la progresión tumoral pero no elimina al tumor. La estabilización de la 
progresión tumoral o la reemergencia de tumores en animales de experimentación y en pacientes, como consecuencia de inmunodeficiencias, son evidencias de esta etapa; y 3) escape, etapa en la que la progresión tumoral no puede ser controlada por el sistema inmune por la emergencia de una gran diversidad mecanismos que afectan la inmunovigilancia. Estos mecanismos incluyen la reducción de la inmunogenicidad de las células neoplásicas, la expresión en membrana y/o secreción al microambiente tumoral de moléculas con efecto inmunosupresor, la alteración de la maduración de las células dendríticas, el incremento en la frecuencia sistémica y/o reclutamiento al microambiente tumoral de células mieloides y linfoides con actividad inmunosupresora, y las alteraciones en el tráfico leucocitario al microambiente tumoral.

Es importante remarcar que el establecimiento de una $\mathrm{u}$ otra estrategia de escape a la inmunovigilancia se debe a la presión selectiva que ejercen los mecanismos efectores del sistema inmune sobre las células neoplásicas. En consecuencia, la manipulación de estos mecanismos de escape como alternativa de inmunoterapia deberá tener un enfoque de precisión, basado no solamente en la caracterización genética del tumor, sino también en la comprensión de los mecanismos de inmunoevasión más relevantes en cada neoplasia ${ }^{(11,12)}$.

\section{INMUNOTERAPIA EN CÁNCER: DE ACTIVAR ACTIVADORES A INHIBIR INHIBIDORES}

Considerando las características de la relación entre el sistema inmune y las células neoplásicas, el cáncer manifiesta una fisiopatología de tres tipos: 1) inmunogénica, es decir, con expresión de antígenos inmunogénicos, activación de las células $\mathrm{T}$ antitumorales e inducción de anticuerpos, lo que marca un pronóstico favorable; 2) inflamatoria, con elevada producción de moléculas inflamatorias, proangiogénicas e inmunosupresoras, así como el incremento en la frecuencia y actividad supresora de células mieloides supresoras (MDSCs, del inglés Myeloid Derived Suppressor Cells) y células $\mathrm{T}$ regulatorias (Treg), que produce una alta agresividad tumoral y pronóstico desfavorable; 3) escapatoria, con pobre activación inmune y producción de citoquinas, de pronóstico intermedio. La inmunoterapia dirigida a cada uno de estos escenarios sería diferente, siendo más efectivo el uso de drogas de bloqueo de puntos de control de la inmunidad, en el tipo inmunogénico; el uso de antiangiogénicos, anticuerpos que bloqueen el factor de crecimiento transformante- $\beta$ y drogas de bloqueo de puntos de control de la inmunidad, en el tipo inflamatorio; y terapia celular y anticuerpos específicos a antígenos tumorales, en el tipo escapatorio $^{(13)}$.

De acuerdo con estos puntos de activación e inhibición señalados, las estrategias fundamentales de la inmunoterapia serían tres: 1) la estimulación de la interacción entre las APC y las células T (ej. vacunas tumorales); 2) la inhibición de señales coinhibitorias (ej. bloqueo de puntos de control de la inmunidad), así como de células inmunosupresoras como las Treg y MDSCs; 3) la transferencia adoptiva de efectores inmunes como anticuerpos monoclonales y células $\mathrm{T}^{(14)}$.

\section{Vacunas tumorales}

Están dirigidas a proveer al sistema inmune del paciente de antígenos tumorales, péptidos o células enteras. Su efectividad es bastante variable ya que depende de que no exista una alta carga tumoral en el paciente y mientras sea posible vencer los mecanismos de tolerancia inmune ${ }^{(14)}$. A pesar de que en estudios iniciales de tipo preclínico los resultados han sido desalentadores, aún existe un marcado interés en el entendimiento del rol de las vacunas tumorales como adyuvantes a otras terapias y reformulando su uso en el tratamiento de distintos tipos de neoplasias malignas ${ }^{(15,16)}$.

\section{Bloqueo de puntos de regulación e inmunomodulación celular}

Los puntos de regulación contribuyen a modular la magnitud de la respuesta inmune de las células $\mathrm{T}$ y son críticas para evitar fenómenos de autoinmunidad. Sin embargo, su función en relación con la terapia dirigida en procesos neoplásicos limita la intensidad y duración de las respuestas antitumorales de manera significativa. Los puntos de regulación claves más estudiados incluyen las moléculas de superficie celular T: CTLA-4, PD-1 y su ligando, Tim-3 (del inglés, T cell inmunoglobulin and mucin domain-containing protein-3) y LAG-3 (del inglés, lymphocyte activation gene-3), las cuales han sido altamente atractivas como moléculas diana en el desarrollo de la inmunoterapia contra el cáncer ${ }^{(17,18)}$.

\section{Inmunoterapia adoptiva}

Se basa en la administración a los pacientes de anticuerpos monoclonales de relevancia en la práctica clínica, como el rituximab (anti-CD20), bevacizumab (antifactor de crecimiento vascular endotelial), cetuximab (antireceptor del Factor de Crecimiento Epidérmico), por solo mencionar algunos de los que ya se encuentran como terapias de primera línea en neoplasias sólidas y hematológicas ${ }^{(17,18)}$. En esta lista se incluyen inmunoconjugados, como el Y-ibritumomab tiuxetan y el I-tositumomab para linfoma, y los anticuerpos biespecíficos, quienes se unen al antígeno tumoral y a la célula inmune efectora, como el blinatumomab (anti-CD19/ anti-CD20). En esta categoría terapéutica se incluye la transferencia de células $\mathrm{T}$ autólogas, las cuales se extraen del paciente para ser modificadas ex vivo, y luego se infunden nuevamente. Las células T-CAR (del inglés, chimeric antigen receptor-T cells) es una de estas variantes celulares que está siendo estudiada de forma exhaustiva debido su gran potencial terapéutico ${ }^{(19)}$. 


\section{POSICIONAMIENTO DE LA INMUNOTERAPIA: DE LA MONOTERAPIA A LA COMBINACIÓN}

El uso combinado de anticuerpos para bloquear PD1/PDL1 y CTLA-4 tiene como racionalidad que ambas señales se involucran en etapas diferentes de la actividad linfocitaria. CTLA-4 funciona regulando la activación de las células T en el ganglio linfático, mientras que PD-1 juega su papel regulador principalmente en los tejidos periféricos. Por tanto, esta combinación permite bloquear señales inhibitorias de la respuesta inmune en diferentes partes o puntos del ciclo de la inmunidad del cáncer, favoreciendo la infiltración de células $\mathrm{T}$ en el tumor y promoviendo la actividad citotóxica de las células $\mathrm{T}$ antitumorales (Figura 2$)^{(20,21)}$. La terapia dual anti CTLA-4/PD-1 modifica el microambiente de un tumor supresor o relativamente no inflamatorio a un tumor inflamatorio. Este efecto dual de los dos agentes permite que se ejerzan mecanismos complementarios y no redundantes con el objetivo de aumentar la tasa de respuesta ${ }^{(22,23)}$.

En la actualidad, los agentes de mayor trascendencia clínica son aquellos que bloquean puntos de control de la inmunidad, como el ipilimumab (anti-CTLA-4), el nivolumab (anti-PD-1) y el pembrolizumab (anti-PD-1) ${ }^{(20,21)}$. El ipilimumab tiene como mecanismo dirigirse a la proteína de superficie de punto de control CTLA-4 que se encuentra en la célula $\mathrm{T}$. El nivolumab y pembrolizumab se dirigen a la proteína de punto de control PD-1 que también se encuentran en las células $\mathrm{T}$. El reconocimiento por los anticuerpos de CTLA-4 o PD-1 evita la unión con sus ligandos CD80/ CD86 y PDL-1/PDL-2, respectivamente, lo que bloquea las señales de inactivación linfocitaria, potenciando la cronicidad del ataque inmunológico a la célula tumoral.

Actualmente, tiene gran trascendencia la combinación de agentes inmunoterapéuticos e incluso la combinación con otros tipos de terapias, como la terapia blanco, la quimioterapia y la radioterapia ${ }^{(23-25)}$. Un buen ejemplo de éxito es el uso de inmunoterapia en melanoma, en donde se ha probado que la terapia combinada es efectiva en estudios preclínicos y estudios clínicos de fase I a III ${ }^{(26-28)}$. Este éxito en melanoma ha motivado numerosos estudios clínicos de combinación entre el bloqueo de la señal PD-1/PDL-1 y de CTLA-4 en diferentes neoplasias, así como con otros puntos de control como TIM-3 y LAG-3 ${ }^{(29-31)}$. El bloqueo del par molecular PD1/PDL-1 es la base de estas combinaciones, debido a su amplio espectro en diversos cánceres y su favorable perfil de toxicidad ${ }^{(20)}$.

Además, se han propuesto otros modelos de los potenciales mecanismos celulares que se producen con la combinación anti-CTLA-4 y anti-PD-1: 1) las mismas células T pueden ser blanco de ambos agentes en el mismo sitio de activación, conduciendo a un bloqueo más efectivo de las señales inhibitorias de la actividad del sistema inmune; 2) diferentes poblaciones de células $\mathrm{T}$ son blanco dentro del mismo sitio de activación, conduciendo a efectos sinérgicos; 3) las mismas células $\mathrm{T}$ son blanco de ambos agentes pero en diferente espacio y tiempo, conduciendo a una señalización más prolongada, y 4) diferentes poblaciones celulares $\mathrm{T}$ son blanco en diferentes tejidos (Figura 3$)^{(32)}$.

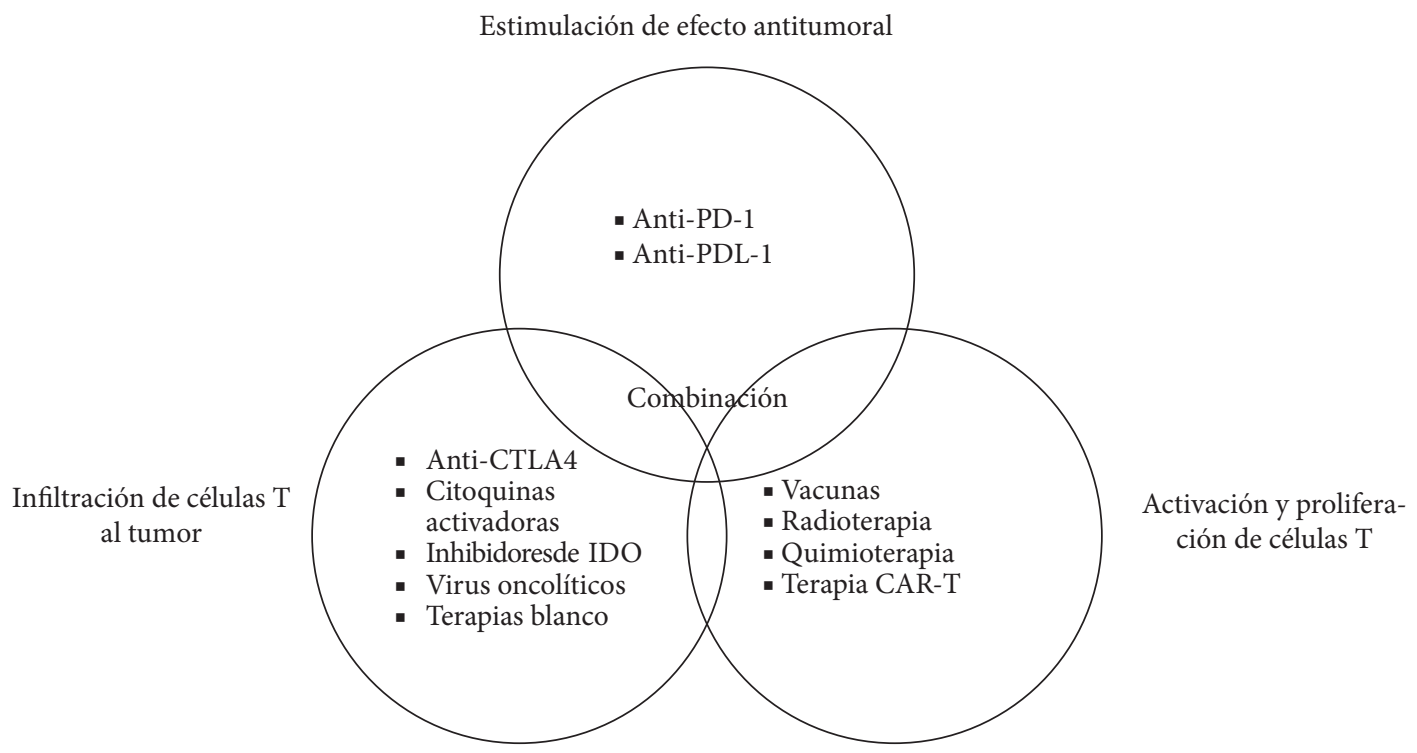

Figura 2. Modelo de combinaciones con inmunoterapia y otros agentes. PD-1: programmed cell death protein 1, PDL-1: Programmed death-ligand 1, CTLA-4: cytotoxic T lymphocyte antigen 4, IDO: indoleamina 2,3-dioxygenasa, CAR-T: chimeric antigen receptor.

Adaptado de Atkins M. Immunotherapy Combinations With Checkpoint Inhibitors in Metastatic Melanoma: Current Approaches and Future Directions. Semin Oncol. 2015 Dec;42 Suppl 3:S12-19. doi: 10.1053/j.seminoncol.2015.10.002 


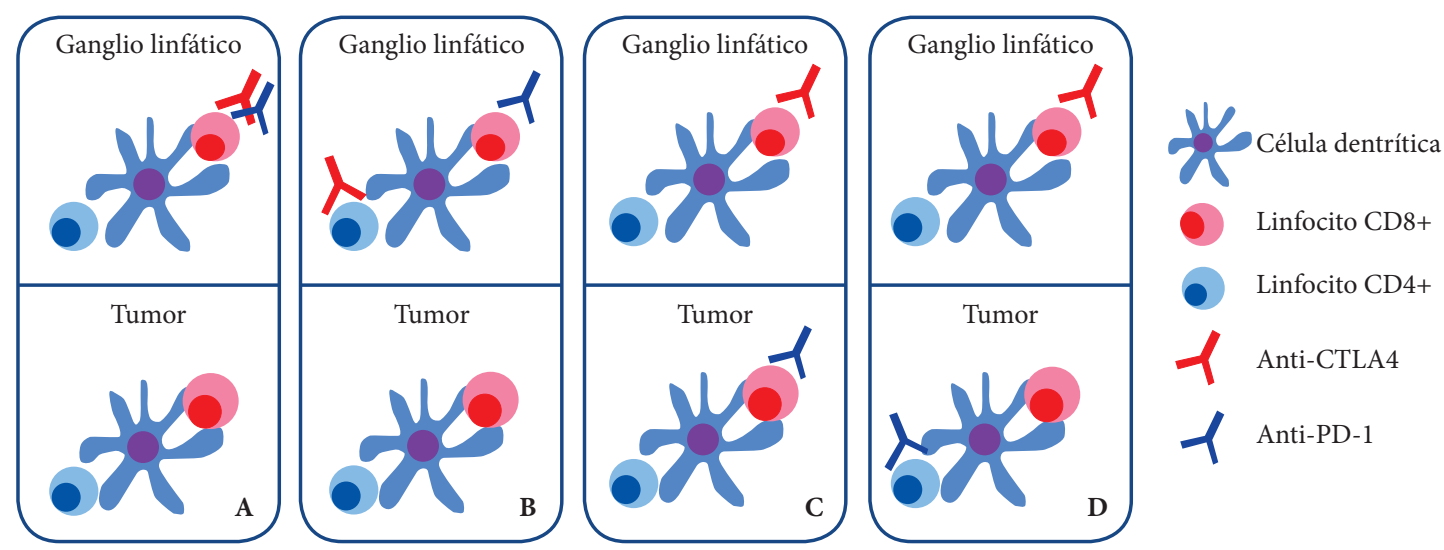

Figura 3. Mecanismos celulares del tratamiento dual con anti-CTLA-4 y anti-PD-1: A. Una misma célula T puede ser blanco de ambos agentes (anti-CTLA-4 y anti-PD-1) en el sitio de activación (ganglios linfáticos), conduciendo a un mayor bloqueo de señales inhibitorias y aumentando la activación del sistema inmune; B. Diferentes poblaciones de células T( CD8+ y CD4+) son blanco en el sitio de activación (provocando efectos sinérgicos; C. Una misma célula T es blanco de ambos agentes pero en diferente espacio y tiempo, conduciendo a una señalización más prolongada; D. Diferentes poblaciones celulares T son blanco de los agentes en diferentes tejidos.

Adaptado de Wei SC, Duffy CR, Allison JP. Fundamental Mechanisms of Immune Checkpoint Blockade Therapy. Cancer Discov. 2018 Sep;8(9):1069-86. doi: 10.1158/2159-8290.CD-18-0367.

\section{RETOS Y PERSPECTIVAS DE LA INMUNO-ONCOLOGÍA}

A pesar de que la inmunoterapia ha significado un gran avance en el tratamiento del cáncer en los últimos 20 años, en algunos tipos de cáncer las tasas de respuesta son menores al $20 \%$, por lo que aún existen más desafíos que alcanzar en esta área ${ }^{(33)}$. Dentro de los retos de la inmunoterapia en cáncer se encuentran:

\section{Estrategia de combinación}

Sobre la base de los inhibidores de los puntos de control de la inmunidad, existen algunas consideraciones en relación con la combinación con los siguientes agentes:

a. Agentes agonistas: la estrategia de combinar un agonista con un inhibidor dependerá de la concomitancia/secuencialidad. Existe evidencia preclínica que muestra que es mejor la estrategia de secuencia que la concomitancia empezando con el agonista y luego el inhibidor. Otro factor para tomar en cuenta es la dosis del agente agonista ${ }^{(34,35)}$.

b. Agentes inhibidores: los resultados son evidentes con la combinación de los anticuerpos monoclonales nivolumab e ipilimumab en cáncer renal y melanoma; sin embargo, los eventos adversos inmunorelacionados se incrementan a toxicidad se incrementa notablemente ${ }^{(36)}$.

c. Quimioterapia: agentes citotóxicos pueden aumentar la exposición de antígenos tumorales y de patrones moleculares asociados a daño (DAMPs, del inglés Danger Associated Molecular Patterns) ${ }^{(36,37)}$, reduciendo además la actividad de las MDSCs y las Treg ${ }^{(38,39)}$. Actualmente, quimioterapia en combinación con pembrolizumab es el estándar en primera línea de cáncer de pulmón de células no pequeñas tipo adenocarcinoma y escamoso. En el cáncer de mama triple negativo, el anticuerpo atezolizumab asociado a nab-paclitaxel en primera línea logra mejorar la sobrevida global fundamentalmente en pacientes cuyas células neoplásicas expresan la molécula PDL-1 ${ }^{(40)}$.

d. Agentes reguladores epigenéticos: diversos mecanismos epigenéticos están involucrados en la resistencia a la inmunoterapia, como el silenciamiento de genes del complejo mayor de histocompatibilidad tipo-I, la producción de citoquinas inmunosupresoras y la expresión de PD-1 ${ }^{(41,42)}$. El empleo de agentes como los inhibidores de desacetilasas de histonas o inhibidores de la metilación del DNA podrían asociarse a agentes de inmunoterapia ${ }^{(43)}$.

\section{Identificación de un biomarcador predictivo}

Entre el 5 y $20 \%$ de los pacientes que no expresan la molécula PDL-1 pueden responder a la terapia immune, ${ }^{(44)}$ lo que hace de la molécula PDL-1 un biomarcador imperfecto. PDL-1 y linfocitos infiltrantes de tumor podrían mejorar su capacidad de predicción ${ }^{(45,46)}$. La evaluación de la carga mutacional, que refleja la cantidad de neoantígenos que pueden ser detectados por el sistema inmune, emerge como una interesante opción por los resultados en cáncer de pulmón de células no pequeñas y melanoma. Ambas malignidades poseen alta tasa de mutaciones inducidas por el tabaquismo y los rayos ultravioleta, respectivamente. La inestabilidad de microsatélite correlaciona con la tasa de mutaciones y la cantidad de neoantígenos.

La caracterización del microbioma intestinal parece identificar a los pacientes que pueden ser respondedores a la inmunoterapia. Dos importantes hechos confirman el rol de la microbiota y la regulación del sistema inmune ligado al control del cáncer: primero, la influencia de los antibióticos sobre la efectividad de la inmunoterapia; segundo, el trasplante de microbiota fecal con Akkermansia muciniphi- 
la aplicado a ratones con cáncer de pulmón de células no pequeñas incrementaría la efectividad de la inmunoterapia. Lo anterior refleja la importancia de la microbiomata en la regulación de la vigilancia inmunológica al cáncer y en la sensiblidad de los pacientes a la inmunoterapia ${ }^{(47-49)}$.

\section{Identificación de blancos moleculares en el microambiente tumoral}

Es reconocida la influencia del microambiente tumoral inmunosupresor en la actividad inmune. Diferentes tipos celulares del inmunocontexto en el microambiente tumoral contribuyen a la progresión del cáncer, como es el caso de los macrófagos M2, los neutrófilos N2, las MDSCs, entre otros. Recientemente un estudio clínico Fase I-b, demostró que el bloqueo de la señal negativa mediada por la molécula CD47 en macrófagos incrementa la fagocitosis de células tumorales, con resultados impresionantes en pacientes con linfoma de células grandes $\mathrm{B}$ difuso y linfoma folicular, multitratados ${ }^{(50)}$. Otra estrategia en desarrollo es el uso de inhibidores farmacológicos de la enzima IDO en tumores. La actividad de esta enzima deprime o disminuye la disponibilidad de triptófano e incrementa la liberación de la molécula kineurina, ambas condiciones nocivas para la actividad linfocitaria ${ }^{(51)}$.

\section{REFERENCIAS BIBLIOGRÁFICAS}

1. Restifo NP, Dudley ME, Rosenberg SA. Adoptive immunotherapy for cancer: harnessing the T cell response. Nat Rev Immunol. 2012;12(4):269-81. doi: $10.1038 /$ nri3191

2. Hanahan D, Weinberg R. Hallmarks of cancer. Cell 2000; 100(1):57-70. doi: 10.1016/s0092-8674(00)81683-9

3. Hanahan D, Weinberg RA. Hallmarks of cancer: the next generation. Cell. 2011;144(5):646-74. doi: 10.1016/j.cell.2011.02.013

4. Ribatti D. The concept of immune surveillance against tumors. The first theories. Oncotarget. 2017;8(4):7175-80. doi: 10.18632/oncotarget.12739

5. Chen DS, Mellman I. Oncology meets immunology: the cancer-immunity cycle. Immunity. 2013;39(1):1-10. doi: 10.1016/j.immuni.2013.07.012

6. Fridman WH, Pagès F, Sautès-Fridman C, Galon J. The immune contexture in human tumours: impact on clinical outcome. Nat Rev Cancer. 2012 15;12(4):298-306. doi: 10.1038/nrc3245

7. Galon J, Mlecnik B, Bindea G, Angell HK, Berger A, Lagorce C, et al. Towards the introduction of the "Immunoscore" in the classification of malignant tumours. J Pathol. 2014;232(2):199-209.doi: 10.1002/path.4287

8. Taube JM, Galon J, Sholl LM, Rodig SJ, Cottrell TR, Giraldo NA, et al. Implications of the tumor immune microenvironment for staging and therapeutics. Mod Pathol. 2018;31(2):214-34. doi: 10.1038/modpathol.2017.156

9. Greaves M, Maley CC. Clonal evolution in cancer. Nature. 2012;481(7381):306-13. doi: 10.1038/nature10762

10. Vesely MD, Kershaw MH, Schreiber RD, Smyth MJ. Natural innate and adaptive immunity to cancer. Annu Rev Immunol. 2011;29:235-71. doi: 10.1146/annurev-immunol-031210-101324

11. MéndezR, Ruiz-Cabello F, Rodríguez T, Del Campo A, Paschen A, SchadendorfD, et al. Identification of different tumor escape mechanisms in several metastases from a melanoma patient undergoing immunotherapy. Cancer Immunol Immunother. 2007;56(1):88-94. doi: 10.1007/s00262-006-0166-2

12. Yuan J, Page DB, Ku GY, Li Y, Mu Z, Ariyan C, et al. Correlation of clinical and immunological data in a metastatic melanoma patient with heterogeneous tumor responses to ipilimumab therapy. Cancer Immun. 2010;10:1.

\section{CONCLUSIONES}

La inmunoterapia combinada ha ganado protagonismo entre los enfoques terapéuticos indicados a pacientes con cáncer. El diseñar y ensayar combinaciones no consiste en la mera adición directa de diferentes agentes, sino que se debe tener en cuenta la complejidad de la biología de tumor, su microambiente y la capacidad de respuesta de cada agente. La inmunooncología ha logrado un éxito sin precedentes con múltiples aprobaciones por la Administración de Drogas y Medicamentos de los Estados Unidos (FDA) para diferentes neoplasias, incluyendo los inhibidores de los puntos de control de la inmunidad y las células T-CAR. Aunque los resultados superan lo alcanzado por la quimioterapia, aún no se puede precisar si estas respuestas duraderas representan remisión a largo plazo.

Contribuciones de autoría: LV, DC, JL y BB han participado en la concepción del artículo. Todos los autores participaron de la redacción del artículo y la aprobación de la versión final.

Fuentes de financiamiento: Autofinanciado.

Conflictos de interés: Los autores declaran no tener conflictos de intereses en la publicación del artículo.
13. Becht E, Giraldo NA, Dieu-Nosjean M-C, Sautès-Fridman C, Fridman WH. Cancer immune contexture and immunotherapy. Curr Opin Immunol. 2016;39:7-13. doi: 10.1016/j.coi.2015.11.009

14. Villegas C, Ramírez D. Las células Treg en la inmunoedición e inflamación asociada al cáncer. Rev Fac Med. 2015;58(6):5-17.

15. Makkouk A, Weiner GJ. Cancer immunotherapy and breaking immune tolerance: new approaches to an old challenge. Cancer Res. 2015;75(1):510. doi: $10.1158 / 0008-5472$.CAN-14-2538

16. Kumai T, Kobayashi H, Harabuchi Y, Celis E. Peptide vaccines in cancer-old concept revisited. Curr Opin Immunol. 2017;45:1-7.doi:10.1016/j. coi.2016.11.001

17. Scott AM, Wolchok JD, Old LJ. Antibody therapy of cancer. Nat Rev Cancer. 2012;12(4):278-87.doi:10.1038/nrc3236

18. Galluzzi L, Vacchelli E, Fridman WH, et al. Trial watch: Monoclonal antibodies in cancer therapy. Oncoimmunology. 2012;1(1):28-37. doi:10.4161/onci.27297

19. Prasad V. Immunotherapy: Tisagenlecleucel - the first approved CART-cell therapy: implications for payers and policy makers. Nat Rev Clin Oncol. 2018;15(1):11-12.doi:10.1038/nrclinonc.2017.156

20. Postow MA, Callahan MK, Wolchok JD. Immune Checkpoint Blockade in Cancer Therapy. J Clin Oncol. 2015;33(17):1974-82.doi:10.1186/ s40425-017-0218-5

21. Khalil DN, Smith EL, Brentjens RJ, Wolchok JD. The future of cancer treatment: immunomodulation, CARs and combination immunotherapy. Nat Rev Clin Oncol. 2016;13(5):273-90.doi:10.1038/nrclinonc.2016.25

22. Atkins M. Immunotherapy Combinations With Checkpoint Inhibitors in Metastatic Melanoma: Current Approaches and Future Directions. Semin Oncol. 2015;42 Suppl 3:S12-19.doi: 10.1053/j.seminoncol.2015.10.002

23. Ott PA, Hodi FS, Kaufman HL, Wigginton JM, Wolchok JD. Combination immunotherapy: a road map. J Immunother Cancer. 2017;5:16. doi:10.1186/s40425-017-0218-5 
24. Harris SJ, Brown J, Lopez J, Yap TA. Immuno-oncology combinations: raising the tail of the survival curve. Cancer Biol Med. 2016;13(2):171-93. doi:10.20892/j.issn.2095-3941.2016.0015

25. Fucà G, de Braud F, Di Nicola M. Immunotherapy-based combinations: an update. Curr Opin Oncol. 2018 Sep;30(5):345-51. doi:10.1097/ CCO.0000000000000466

26. Curran MA, Montalvo W, Yagita H, Allison JP. PD-1 and CTLA-4 combination blockade expands infiltrating $\mathrm{T}$ cells and reduces regulatory $\mathrm{T}$ and myeloid cells within B16 melanoma tumors. Proc Natl Acad Sci USA. 2010;107(9):4275-80. doi:10.1073/pnas.0915174107

27. Larkin J, Chiarion-Sileni V, Gonzalez R, Grob JJ, Cowey CL, Lao CD, et al. Combined Nivolumab and Ipilimumab or Monotherapy in Untreated Melanoma. N Engl J Med. 2015;373(1):23-34. doi:10.1056/NEJMoa1504030

28. WolchokJD, Chiarion-Sileni V, Gonzalez R, Rutkowski P, Grob J-J, Cowey CL, et al. Overall Survival with Combined Nivolumab and Ipilimumab in Advanced Melanoma. N Engl J Med. 2017 05;377(14):1345-56. doi10.1056/NEJMoa1709684

29. Marmarelis ME, Aggarwal C. Combination Immunotherapy in Non-small Cell Lung Cancer. Curr Oncol Rep. 2018;20(7):55. doi:10.1007/s11912-018-0697-7

30. Hoffman-Censits J, Wilde L. Combination immunotherapy in genitourinary malignancies. Curr Opin Urol. 2016;26(6):523-8. doi:10.1097/ MOU.0000000000000336

31. George S, Rini BI, Hammers HJ. Emerging Role of Combination Immunotherapy in the First-line Treatment of Advanced Renal Cell Carcinoma: A Review. JAMA Oncol. 2019;5(3):411-421. doi: 10.1001/ jamaoncol.2018.4604

32. Wei SC, Duffy CR, Allison JP. Fundamental Mechanisms of Immune Checkpoint Blockade Therapy. Cancer Discov. 2018;8(9):1069-86. doi:10.1158/2159-8290.CD-18-0367

33. Marshall HT, Djamgoz MBA. Immuno-Oncology: Emerging Targets and Combination Therapies. Front Oncol. 2018;8:315. doi:10.3389/ fonc. 2018.00315

34. Shrimali RK, Ahmad S, Verma V, Zeng P, Ananth S, Gaur P, et. al. Concurrent PD-1 blockade negates the effects of OX40 agonist antibody in combination immunotherapy through inducing T-cell apoptosis. Cancer Immunol Res 2017; 5(9):755-766. doi:10.1158/2326-6066.CIR-17-0292

35. Messenheimer DJ, Jensen SM, Afentoulis ME, Wegmann KW, Feng Z, Friedman DJ, et al. Timing of PD-1 Blockade Is Critical to Effective Combination Immunotherapy with Anti-OX40. Clin Cancer Res. 2017;23(20):6165-77. doi:10.1158/1078-0432.CCR-16-2677

36. Zhou S, Khanal S, Zhang H. Risk of immune-related adverse events associated with ipilimumab-plus-nivolumab and nivolumab therapy in cancer patients. Ther Clin RiskManag. 2019;15:211-221.doi:10.2147/TCRM.S193338

37. Zitvogel L, Kepp O, Kroemer G. Immune parameters affecting the efficacy of chemotherapeutic regimens. Nat Rev Clin Oncol. 2011 Mar;8(3):15160. doi:10.1038/nrclinonc.2010.223

38. Zitvogel L, Galluzzi L, Smyth MJ, Kroemer G. Mechanism of action of conventional and targeted anticancer therapies: reinstating immu- nosurveillance. Immunity. 2013 Jul 25;39(1):74-88.doi: 10.1016/j. immuni.2013.06.014

39. van der Most RG, Currie AJ, Mahendran S, Prosser A, Darabi A, Robinson BWS, et al. Tumor eradication after cyclophosphamide depends on concurrent depletion of regulatory T cells: a role for cycling TNFR2-expressing effector-suppressor T cells in limiting effective chemotherapy. Cancer Immunol Immunother. 2009;58(8):1219-28. doi:10.1007/s00262-008-0628-9

40. Schmid P, Adams S, Rugo HS, Schneeweiss A, Barrios CH, Iwata H, et al. Atezolizumab and Nab-Paclitaxel in Advanced Triple-Negative Breast Cancer. N Engl J Med. 2018;379(22):2108-21. doi: 10.1056/ NEJMoa1809615

41. Chang C-C, Ferrone S. Immune selective pressure and HLA class I antigen defects in malignant lesions. Cancer Immunol Immunother. 2007;56(2):227-36. doi:10.1007/s00262-006-0183-1

42. Yasmin R, Siraj S, Hassan A, Khan AR, Abbasi R, Ahmad N. Epigenetic regulation of inflammatory cytokines and associated genes in human malignancies. Mediators Inflamm. 2015;2015:201703.doi: 10.1155/2015/201703

43. Wrangle J, Wang W, Koch A, Easwaran H, Mohammad HP, Vendetti F, et al. Alterations of immune response of Non-Small Cell Lung Cancer with Azacytidine. Oncotarget. 2013;4(11):2067-79. doi:10.18632/ oncotarget.1542

44. Fusi A, Festino L, Botti G et al. PD-L1 expression as a potential predictive biomarker. Lancet. Oncol. 2015; 16(13):1285-7. doi:10.1016/ S1470-2045(15)00307-1

45. Smyth MJ, Ngiow SF, Ribas A, Teng MWL. Combination cancer immunotherapies tailored to the tumour microenvironment. Nat Rev Clin Oncol. 2016;13(3):143-58. doi:10.1038/nrclinonc.2015.209

46. Teng MWL, Ngiow SF, Ribas A, Smyth MJ. Classifying Cancers Based on T-cell Infiltration and PD-L1. Cancer Res. 2015;75(11):2139-45. doi:10.1158/0008-5472.CAN-15-0255

47. Routy B, Le Chatelier E, Derosa L, Duong CPM, Alou MT, Daillère R, et al. Gut microbiome influences efficacy of PD-1-based immunotherapy against epithelial tumors. Science. 2018;359(6371):91-7. doi:10.1126/ science.aan 3706

48. Gopalakrishnan V, Spencer CN, Nezi L, Reuben A, Andrews MC, Karpinets TV, et al. Gut microbiome modulates response to anti-PD-1 immunotherapy in melanoma patients. Science. 2018;359(6371):97-103. doi:10.1126/science.aan4236

49. Matson V, Fessler J, Bao R Chongsuwat T, Zha Y, Alegre ML, et. al. The commensal microbiome is associated with anti-PD-1 efficacy in metastatic melanoma patients. Science 2018; 359(6371):104-108. doi:10.1126/ science.aao3290

50. Advani R, Flinn I, Popplewell L, Forero A, Bartlett NL, Ghosh N, et al. CD47 Blockade by Hu5F9-G4 and Rituximab in Non-Hodgkin's Lymphoma. N Engl J Med. 2018;379(18):1711-21.doi: 10.1056/NEJMoa1807315

51. Epacadostat Shows Value in Two SCCHN Trials. Cancer Discov. 2017;7(9):OF2. doi: 10.1158/2159-8290.CD-NB2017-100 